\title{
Gestão do conhecimento na área de saúde: compartilhamento e aprendizagem
}

\begin{abstract}
RESUMO | Objetivo: identificar a Gestão do Conhecimento da saúde. Método: Realizou-se um estudo de revisão integrativa, com base nas recomendações do método PRISMA para mapear processos, abordagens, técnicas, procedimentos e instrumentos de pesquisa sobre a GC na área da saúde. Foram incluídos trabalhos nacionais e internacionais em português e inglês a partir de 2016 até 2020 Resultado: Foram identificados 103 artigos nas bases de buscas e após análise criteriosa, incluídos nove para compor os resultados e discussão. Conclusão: O importante na área de saúde são os processos e as estratégias da gestão do conhecimento serem baseados em modelos já comprovados e eficazes, além de abordados de forma sistemática e estruturada. Os profissionais de saúde possuem características específicas que os habilitam a agir de maneira coordenada e colaborativa. Ressalta-se que, para que o compartilhamento do conhecimento, deve existir confiança mútua e respeito.

Descritores: Gestão do conhecimento; Aprendizagem; Capacitação; Saúde; Qualificação.
\end{abstract}

ABSTRACT | Objective: to identify Health Knowledge Management. Method: An integrative review study was carried out, based on the recommendations of the PRISMA method to map research processes, approaches, techniques, procedures and instruments on KM in the health area. National and international works in Portuguese and English were included from 2016 to 2020 Result: 103 articles were identified in the search bases and after careful analysis, eight were included to compose the results and discussion. Conclusion: The important thing in the health area is that knowledge management processes and strategies are based on proven and effective models, in addition to being approached in a systematic and structured way. Health professionals have specific characteristics that enable them to act in a coordinated and collaborative way. It is noteworthy that, for knowledge sharing, there must be mutual trust and respect.

Descriptors: Knowledge management; Learning; Training; Health; Qualification.

RESUMEN | Objetivo: identificar la Gestión del Conocimiento en Salud. Método: Se realizó un estudio de revisión integradora, con base en las recomendaciones del método PRISMA para mapear procesos, enfoques, técnicas, procedimientos e instrumentos de investigación sobre la GC en el área de la salud. Se incluyeron trabajos nacionales e internacionales en portugués e inglés de 2016 a 2020. Resultado: Se identificaron 103 artículos en las bases de búsqueda y luego de un análisis cuidadoso, se incluyeron ocho para componer los resultados y la discusión. Conclusión: Lo importante en el área de la salud es que los procesos y estrategias de gestión del conocimiento se basan en modelos probados y efectivos, además de ser abordados de manera sistemática y estructurada. Los profesionales de la salud tienen características específicas que les permiten actuar de forma coordinada y colaborativa. Cabe señalar que, para compartir conocimientos, debe haber confianza y respeto mutuos

Descriptores: Gestión del conocimiento; Aprendiendo; Capacitación; Salud; Calificación.

\section{Bruno Gonçalves da Silva}

Enfermeiro (UNIFENAS), Mestre em Administração de Empresas. (Centro Universitário UNA), Doutorando em Sistemas de Informações e Gestão do Conhecimento (Universidade FUMEC).

ORCID: 0000- 0001-5173-0036

\section{Vânia Paula de Carvalho}

Enfermeira (PUC-MINAS), Enfermeira Intensivista (IEC-PUC MG) e Enfermeira Aeroespacial (FIC-UNIVIRTUAL), Mestre em Promoção da Saúde e Prevenção da Violência (UFMG).

ORCID: 0000-0002-9336-3606

\section{Maria Eduarda Becho Arger Marchetti}

Especialista em Anestesiologista e Medicina Aeroespacial

ORCID: 0000-0002-0242-243X

\section{André Alves Elias}

Médico (UNIFENAS) Médico de Bordo na Unimed Aeromédica e BOA-SAMU BH ORCID: 0000-0002-1103-6450

\section{Flávio Lopes Ferreira}

Mestre em Fisiologia e Farmacologia (UFMG), Cirurgião Geral e Especialista em Medicina Aeroespacial

ORCID: 0000-0001-7740-4394

\section{Armando Sérgio de Aguiar Filho}

Doutor em Gestão da Informação e do Conhecimento (UFMG).

ORCID: 0000-0001-5542-7165

Recebido em: 27/08/2021

Aprovado em: 30/11/2021
INTRODUÇÃO

A Gestão do Conhecimento (GC) ao ser implementada nas empresas é percebida como uma tarefa difícil e complexa, apesar de suas inúmeras e percebidas vantagens. Como resultado, muitas organizações não conseguem processá-la com sucesso, pois o conhecimento possui um valor para o sucesso dos negócios e que, quando bem gerido, tende a trazer vantagem e ser cada vez mais necessário para a sobrevivência organizacional.1

Sua relevância situa-se na constatação de que, em qualquer contexto, é fundamental se considerar a complexidade do ser humano, teoria fundamentada pelo antropólogo, sociólogo e filósofo Edgar Morin, pois "toda e 
qualquer unidade de comportamento humano (práxica) é, ao mesmo tempo, genética / cerebral / social / cultural / ecossistêmica". Disso decorre que "o todo tem um certo número de qualidades e de propriedades que não aparecem nas partes quando elas se encontram separadas". Por consequência, "o conhecimento das partes constituintes não basta para o conhecimento do todo, e o conhecimento do todo, claro, não pode ser isolado do conhecimento das partes", especialmente na área da saúde. 2-3

Neste âmbito, a Gestão do Conhecimento poderá favorecer as instituições para que transformem os dados coletados na práxis cotidiana, compartilhe os conhecimentos assimilados durante a jornada profissional e otimize os cuidados em saúde. 1

Para este esclarecimento, este artigo objetiva identificar a Gestão do Conhecimento da saúde. Estabeleceu-se como problema de pesquisa a seguinte indagação: de que forma a Gestão do Conhecimento tem sido pesquisada e quais suas contribuições à área de saúde?

\section{METODOLOGIA}

Para atender o objetivo deste estudo de identificar as bases e as contribuições teóricas, realizou-se um estudo de revisão integrativa da literatura, realizada com base nas recomendações do Preferred Reporting Items for Systematic Reviews and Meta-Analyses (PRISMA) 4 para mapear processos, abordagens, técnicas, procedimentos e instrumentos de pesquisa sobre a GC na área da saúde.

Os critérios de inclusão foram trabalhos publicados em pesquisas / periódicos nacionais e internacionais em português e inglês a partir de 2016 até 2020 e os que apresentaram contribuições a respeito da temática saúde, GC, compartilhamento do conhecimento, aprendizagem em GC e GC em equipes de saúde. Ainda, os critérios de exclusão desconsideraram as publicações prévias a 2016, os duplicados, as que não apresentaram os critérios de inclusão, as que apresentavam títulos e resulecionados e as que não apresentaram adequado nível de evidência.

As publicações que compuseram a amostra foram encontradas nas bases de dados relacionadas na Biblioteca Virtual em Saúde (Bireme) e na Web of Science. Para o acesso, foram utilizadas as seguintes palavras-chave: 'gestão do conhecimento', 'knowledge management', 'compartilhamento do conhecimento', 'knowledge sharing', 'aprendizagem em gestão do conhecimento', 'knowledge management learning' e 'gestão do conhecimento na área da saúde', 'healthcare knowledge mos que não continham os critérios se-

management'.

\section{RESULTADOS}

O total de 103 obras foram identificadas durante a busca inicial das bases de dados. Após a remoção das duplicatas $(n=18)$, foram lidos 85 títulos e resumos. Após seleção de 53 artigos, foram excluídos 41 e incluído 9 artigos finais. O processo de mapeamento, seleção, inclusão e exclusão dos estudos está descrito no fluxograma apresentado na figura 1.

Sobre os oito trabalhos selecionados, no quadro 1 apresenta a síntese sobre suas respectivas características.

Da amostra dos artigos, tem-se uma dissertação de mestrado (11\%) e restante artigos publicados em periódicos nacionais $(55 \%)$ e os internacionais

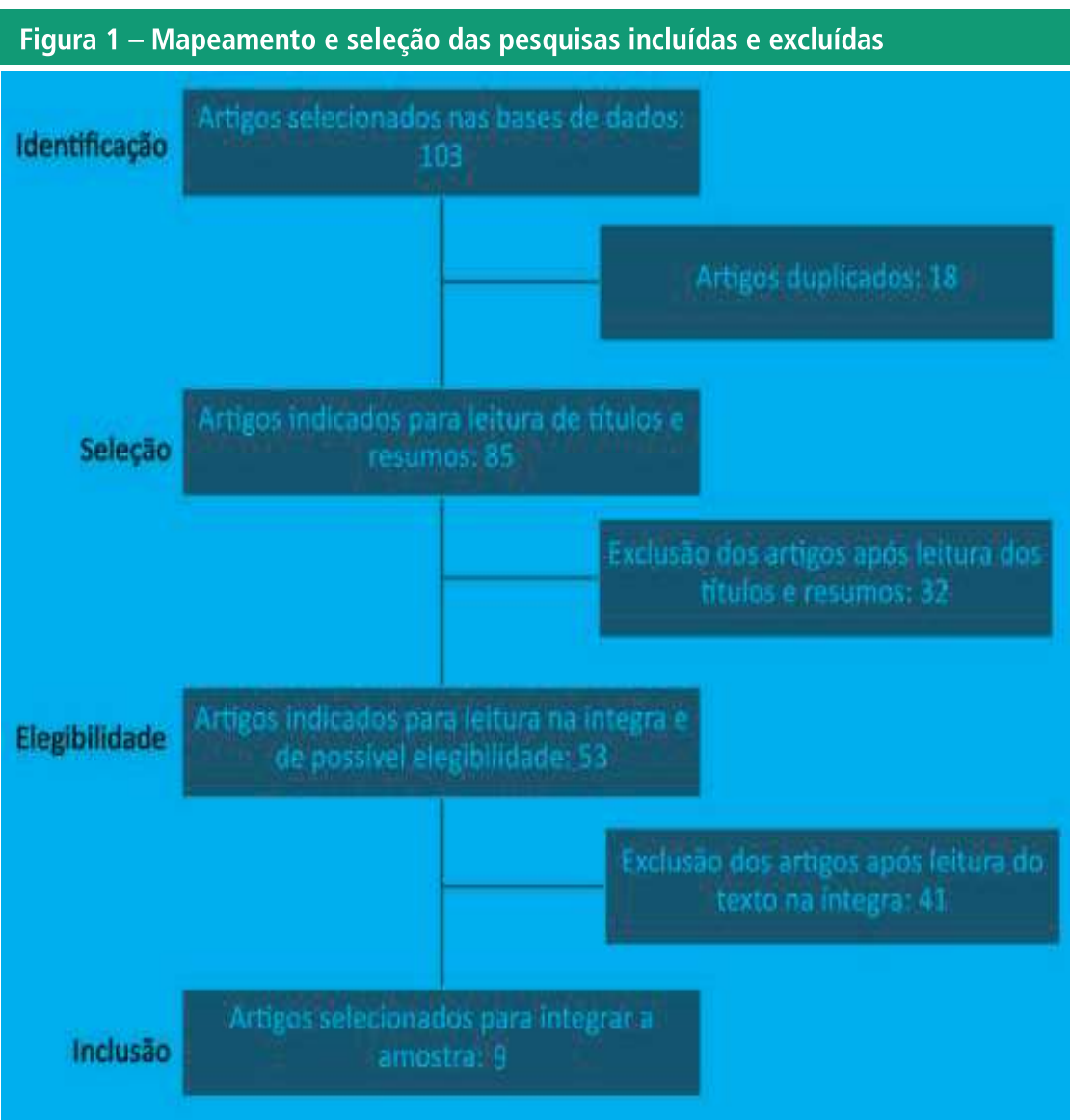

Fonte: Adaptado pelos autores, 2021. 
(34\%). Quanto ao tipo de estudo, têm-se qualitativo $(34 \%)$, retrospectivo observacional $(22 \%)$, quantitativo $(22 \%)$, editorial $(11 \%)$ e revisão sistemática (11\%).

\section{DISCUSSÃO}

Neste contexto, é perceptível a interação do compartilhamento de informações que geram o conhecimento e posteriormente a sua gestão, através de alguns estudos, como por exemplo: educação para apoio a resgate aéreo, 5 percepção de colaboradores de instituições de saúde portuguesas, 6 desenvolvimentos de um modelo de sucesso de sistemas de GC para organizações de saúde neozelandesas, 7 implementações de GC e ferramentas utilizadas para tomadas de decisão nos cuidados à saúde,8 identificação da vantagem competitiva situada na GC direcionada a estratégias de gestão na saúde, 9 identificação de diferentes modelos de GC em hospitais, 10 implementação de GC e ferramentas utilizadas para tomadas de decisão nos cuidados à saúde.

Na área da saúde a Gestão do Conhecimento (GC) é um processo complexo e que apresenta dificuldades de compartilhamento do conhecimento. Morin3 afirma que o conhecimento das partes constituintes não basta para o conhecimento do todo, foco essencialmente administrativo, uma gestão que lida com a recuperação de problemas da saúde das pessoas demanda competências e habilidades mais sensíveis. Neste âmbito, a GC é uma disciplina interessada em favorecer organizações no intuito de transformar múltiplos dados médicos em informações clínicas relevantes e promover o compartilhamento de conhecimento adquirido ao longo de anos de experiências entre os profissionais dos cuidados à saúde. 3

A pesquisa de Ali et al.7 envolveu 263 médicos, 100 atuando em Hamilton e 163 em Wellington, Nova Zelândia. Apresentaram o modelo dos

Quadro 1 - Síntese das características dos artigos selecionados

\begin{tabular}{|c|c|c|c|c|}
\hline $\begin{array}{l}\mathrm{N}^{\circ} \text { do } \\
\text { Artigo }\end{array}$ & Título & Ano & Periódico & $\begin{array}{l}\text { Tipo de } \\
\text { estudo }\end{array}$ \\
\hline $\begin{array}{l}\text { A1 } \\
(5)\end{array}$ & $\begin{array}{l}\text { Estratégia de educação para o apoio aero- } \\
\text { médico: um estudo descritivo. Dissertação }\end{array}$ & 2016 & UFF & Qualitativo \\
\hline $\begin{array}{l}A 2 \\
(6)\end{array}$ & $\begin{array}{l}\text { Gestão do conhecimento em instituições de } \\
\text { saúde portuguesas }\end{array}$ & 2016 & $\begin{array}{l}\text { Revista } \\
\text { Brasileira de } \\
\text { Enfermagem }\end{array}$ & Quantitativo \\
\hline $\begin{array}{l}\mathrm{A} 3 \\
(7)\end{array}$ & $\begin{array}{l}\text { Knowledge management systems success in } \\
\text { healthcare: leadership matters }\end{array}$ & 2017 & $\begin{array}{l}\text { International } \\
\text { Journal of } \\
\text { Medical } \\
\text { Informatics }\end{array}$ & Qualitativo \\
\hline $\begin{array}{l}\text { A4 } \\
(8)\end{array}$ & $\begin{array}{c}\text { Knowledge Management Implementation } \\
\text { and the Tools Utilized in Healthcare for Evi- } \\
\text { dence-Based Decision Making: A Systematic } \\
\text { Review. }\end{array}$ & 2017 & $\begin{array}{c}\text { Journal of } \\
\text { Health Science }\end{array}$ & $\begin{array}{l}\text { Revisão } \\
\text { Sistemática }\end{array}$ \\
\hline $\begin{array}{l}\text { A5 } \\
(9)\end{array}$ & $\begin{array}{l}\text { Effective Knowledge Management Model } \\
\text { (KM) for Healthcare Integrating Success Fac- } \\
\text { tors and Knowledge Management Strategy }\end{array}$ & 2016 & $\begin{array}{l}\text { Asian Hospital } \\
\text { \& Healthcare } \\
\text { Management. }\end{array}$ & $\begin{array}{l}\text { Retrospectivo } \\
\text { observaciona }\end{array}$ \\
\hline $\begin{array}{l}\text { A6 } \\
(10)\end{array}$ & $\begin{array}{l}\text { Gestão do Conhecimento em hospitais } \\
\text { públicos com diferentes modelos de gestão: } \\
\text { alguns fatores explicativos }\end{array}$ & 2017 & $\begin{array}{l}\text { Revista } \\
\text { Brasileira } \\
\text { de Gestão e } \\
\text { Inovação }\end{array}$ & Quantitativo \\
\hline $\begin{array}{l}\text { A7 } \\
(11)\end{array}$ & $\begin{array}{l}\text { Preparação do enfermeiro para o atendi- } \\
\text { mento í múltiplas ví } \text {-timas no resgate aéreo }\end{array}$ & 2021 & $\begin{array}{l}\text { Nursing (São } \\
\text { Paulo) }\end{array}$ & Qualitativo \\
\hline $\begin{array}{l}\text { A8 } \\
(12)\end{array}$ & $\begin{array}{c}\text { Paradoxes of knowledge, management } \\
\text { and knowledge management in Africa: an } \\
\text { editorial introduction. Journal of Knowledge } \\
\text { Management }\end{array}$ & 2020 & $\begin{array}{l}\text { Journal of } \\
\text { Knowledge } \\
\text { Management }\end{array}$ & Editorial \\
\hline $\begin{array}{l}\text { A9 } \\
(13)\end{array}$ & $\begin{array}{l}\text { Knowledge management in the software } \\
\text { industry: how Scrum activities support a } \\
\text { knowledge management cycle }\end{array}$ & 2020 & NAVUS & $\begin{array}{l}\text { Retrospectivo } \\
\text { observaciona }\end{array}$ \\
\hline
\end{tabular}

Fonte: Adaptado pelos autores, 2021.

sistemas de gestão do conhecimento para cuidados de saúde considerando a ampliação de um modelo de sistemas de gestão de conhecimento genéricos existentes, incorporando fatores organizacionais e de sistemas, com o uso de sistemas de gestão de conhecimentos para compartilhamento e para recuperação de variáveis de sucesso utilizadas.

Foram identificados como focos principais da pesquisa de Shahmoradi, Safadari e Jimma8 a implantação de GC na área da saúde, ferramentas GC em saúde, as oportunidades disponíveis e as barreiras existentes. Buscaram proporcionar o conhecimento certo no momento certo, ou seja, no ponto de tomada de decisão pela implementação da GC na área da saúde, entendida como primordial. Para isso, o uso de uma ferramenta adequada para gerenciar o conhecimento e o sistema fácil de usar são importantes requisitos, pois podem melhorar significativamente a qualidade e a segurança dos cuidados prestados aos pacientes.

Para Cruz e Ferreira, 10 a implementação e o próprio sucesso dos programas de GC nas organizações de saúde não ocorrem de forma pacífica, sendo condicionados por um vasto leque de 
fatores, particularmente no modelo de gestão e no número de colaboradores. As autoras analisaram posicionamentos de 671 colaboradores de dez instituições de saúde portuguesas, com diferentes modelos de gestão. Um dos desafios percebidos foi a retenção dos colaboradores e, como consequência, o oferecimento de oportunidades de crescimento e desenvolvimento em troca do seu compromisso para com os objetivos da organização, pois é nas pessoas que reside o principal diferencial competitivo das organizações. Constataram, também, que as práticas de gestão do conhecimento vigentes são ainda pouco consistentes e, na sua maioria, não formalizadas.

A ideia para o desenvolvimento da pesquisa de Bonin5 foi construída a partir dos encontros de trabalho que ocorrem diariamente no Grupamento de Operações Aéreas do Corpo de Bombeiros do Estado do Rio de Janeiro. Foi possível identificar entre as falas dos profissionais da unidade a fragilidade existente nos critérios de acionamento do transporte aeromédico e o desconhecimento dos profissionais de outras unidades em relação ao processo. O pesquisador criou dois fluxogramas, um voltado a um cenário controlado ou com uma única vítima e o outro envolvendo o Incidente com múltiplas vítimas.

O estudo acerca do atendimento a múltiplas vítimas no ambiente aéreo corrobora com o pesquisador citado anteriormente, pois conclui-se que os desafios associados ao ambiente de trabalho, alinhados a capacitação de toda equipe otimizará a segurança e a assistência ao paciente criticamente enfermo.11

O modelo proposto por Ali9 indica ser fundamental que as organizações de saúde incorporem processos, estratégias e fatores de sucesso de GC em seu sistema para a implementação bem-sucedida da GC na área da saúde. Para a pesquisadora, o importante é

que os processos e as estratégias de GC sejam abordados de forma sistemática e estruturada.

O estudo acerca
do atendimento a múltiplas vítimas no ambiente aéreo corrobora com o pesquisador citado anteriormente, pois conclui-se que os desafios associados ao ambiente de trabalho, alinhados a capacitação de toda equipe otimizará a segurança e a assistência ao paciente criticamente enfermo

Na área da saúde, a GC se interessa em transformar múltiplos dados médicos em informações clínicas relevantes e promover o compartilhamento de conhecimento adquirido ao longo de anos de experiências entre os profissionais dos cuidados à saúde, mas a literatura confirma a existência de obstáculos, os quais são determinados pela natureza da instituição, pelo tipo de processo de negócio, de produtos e de clientes.

Neste contexto, os estudos comprovam que a cultura organizacional e do ambiente influenciam, envolvem as pessoas, os sistemas, os processos e por consequência, a gestão do conhecimento12,13. Ressalta-se que, para que o compartilhamento do conhecimento seja produtivo, deve ser fundamentado em confiança mútua e respeito.

\section{CONCLUSÃO}

Conclui-se que, o importante na área de saúde são os processos, as estratégias de Gestão do Conhecimento serem baseados em modelos já comprovados e eficazes. Além disso, que sejam abordados de forma sistemática e estruturada ao contexto sensível, especificamente. Pois, os profissionais desta área possuem características específicas que os habilitam a agir de maneira coordenada e colaborativa, mas podem se tornar mais eficazes se contarem com lideranças transformadoras. Sobremodo, resulte em eficácia e sucesso de atendimento para as suas organizações.

De forma geral, há décadas, as pesquisas apontam que na área de saúde a gestão do conhecimento é um tema fluido. Faz-se necessário a realização de novos estudos que abarquem a Gestão do Conhecimento.

Como limitações do estudo, é possível verificar que a saúde possui diversas especificidades devido ao grande número de profissões e especialidades. Por isso, sugere-se novos estudos para preenchimento das lacunas e especificidades das diversas áreas que o campo da saúde possui. 


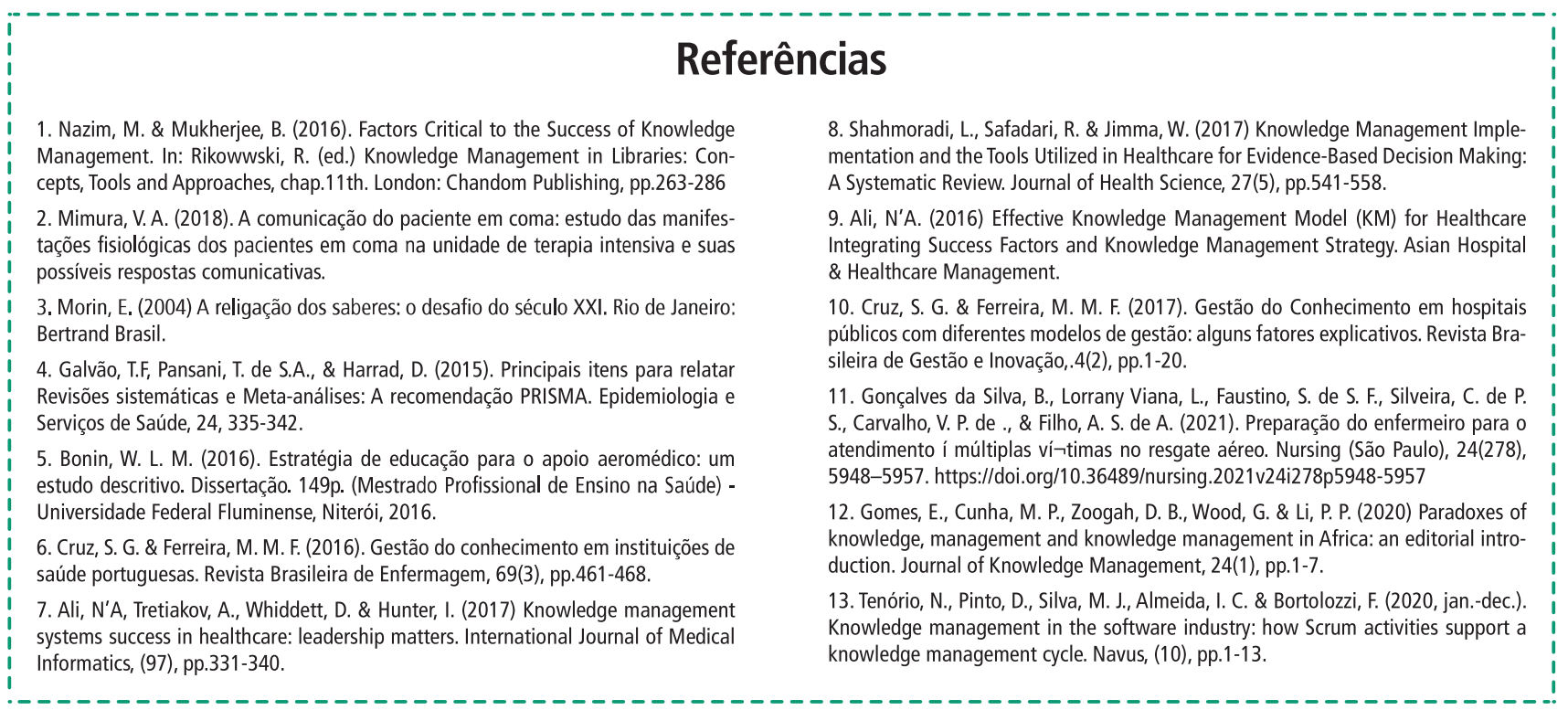

